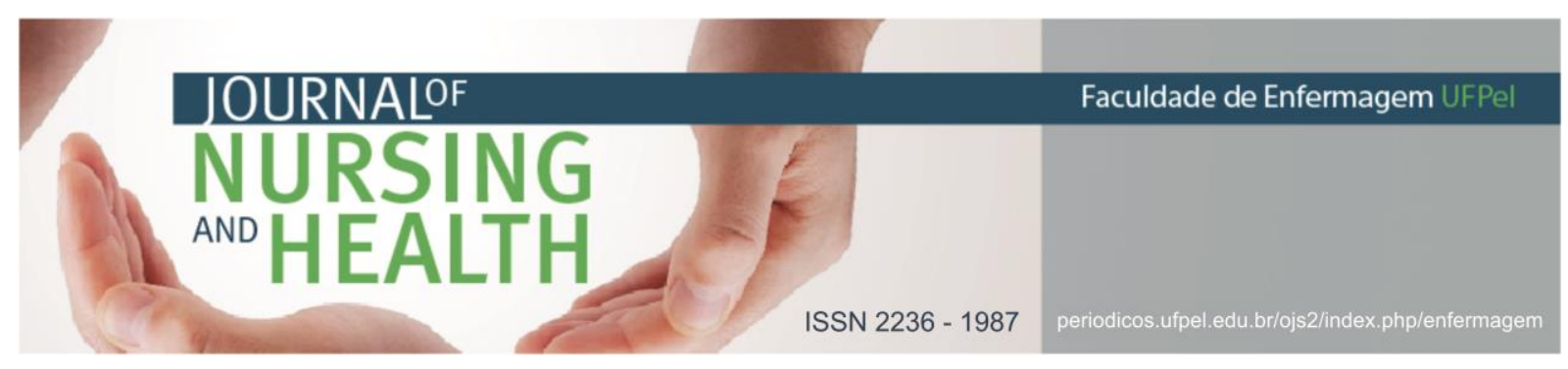

ARTIGO DE REVISÃO

\title{
Avaliação em saúde nos serviços de atenção primária no Brasil: revisão integrativa da literatura
}

\author{
Health evaluation in primary health care in Brazil: integrative review of the \\ literature
Evaluación en salud en los servicios de atención primaria en Brasil: revisión integradora

Nicola, Tainá1; Pelegrini, Alisia Helena Weis²

\begin{abstract}
RESUMO
Objetivo: conhecer e sintetizar a produção científica sobre avaliação em saúde nos serviços de Atenção Primária à Saúde no Brasil. Métodos: trata-se de uma revisão integrativa da literatura realizada por meio de buscas nas bases de dados SciELO, LILACS e MEDLINE, em abril de 2017, utilizando como descritores: Avaliação em Saúde e Atenção Primária à Saúde. Resultados: fizeram parte da pesquisa 13 estudos realizados no Brasil. Foram elaboradas as categorias temáticas: Uso de instrumentos para Avaliação em Saúde e Avaliação do desempenho da Atenção Primária à Saúde. Considerações Finais: a avaliação em saúde é essencial para mensurar o desempenho do sistema de saúde, sendo uma ferramenta condutora de mudanças nos processos de atenção à saúde. 0 instrumento mais utilizado para avaliar é o PCATool. Destaca-se a Estratégia Saúde da Família como o modelo que apresenta melhores resultados nos atributos da Atenção Primária e no desempenho geral da qualidade da atenção.

Descritores: Avaliação em saúde; Atenção primária à saúde; Avaliação de programas e projetos de saúde.
\end{abstract}

\begin{abstract}
Objective: to know and synthesize the scientific production on health evaluation in Primary Health Care services in Brazil. Methods: this is an integrative review of the literature by means of searches in the SCIELO, LILACS and MEDLINE databases, in April 2017, using as descriptors: Health Evalution and Primary Health Care. Results: 13 studies were found conducted in Brazil. Thematic categories were elaborated: Use of instruments for Health Evalution and Evaluation of Primary Health Care performance. Final Considerations: health evaluation is essential to measure the performance of the health system, being a conducive tool for changes in health care processes. The most used instrument to evaluate is PCATool. The Family Health Strategy is highlighted as the model that presents the best results regarding the attributes of Primary Health Care and the overall performance of quality of care.

Descriptors: Health evaluation; Primary health care; Program evaluation.
\end{abstract}

\footnotetext{
${ }^{1}$ Enfermeira. Especialista em Saúde da Família. Universidade Federal de Ciências da Saúde de Porto Alegre. Email: tainanicola@gmail.com https://orcid.org/0000-0002-5966-582X

2 Enfermeira. Doutora em Enfermagem. Universidade Federal de Ciências da Saúde de Porto Alegre. E-mail: alisia@ufcspa.edu.br https://orcid.org/0000-0003-4830-4583
} 


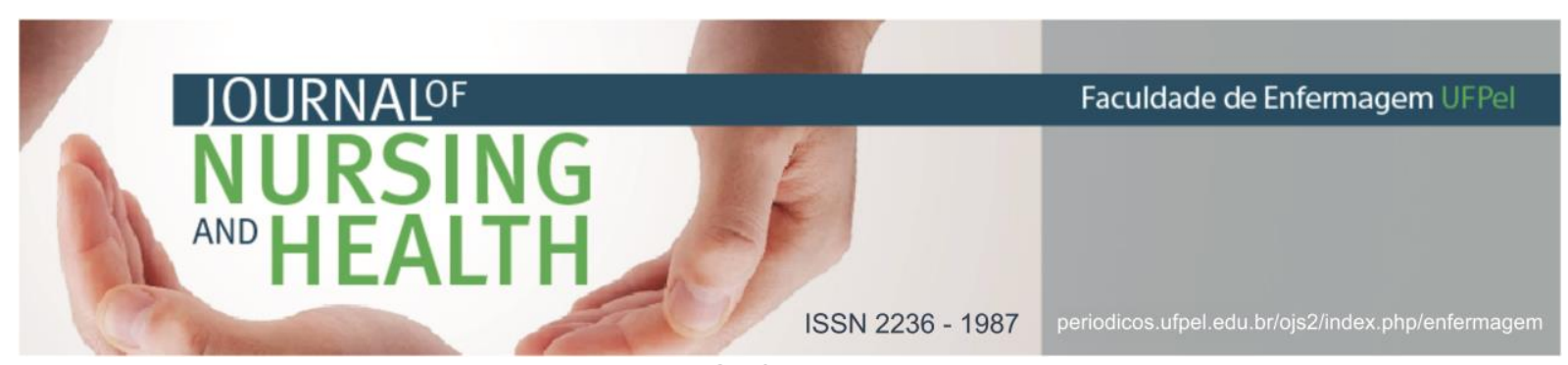

RESUMEN

Objetivo: conocer y sintetizar la literatura científica sobre la evaluación en salud en los servicios de atención primaria de salud en Brasil. Métodos: se trata de una revisión integradora de la literatura realizada por la búsqueda de bases de datos SCIELO, LILACS y MEDLINE, en abril de 2017. Resultados: 13 estudios eran parte de investigación llevados a cabo en Brasil. Los temas se elaboraron: Uso de herramientas para la Evaluación de la Salud y La evaluación de los resultados de la atención primaria. Consideraciones finales: una evaluación de la salud es esencial para medir el desempeño del sistema de salud, y una herramienta conductora cambia en el proceso de atención de la salud. La herramienta más comúnmente utilizada para evaluar es la PCATool. Salud de la Familia es el modelo con los mejores resultados que los atributos de la APS y el rendimiento global de la calidad de la atención.

Descriptores: Evaluación en salud; Atención primaria de salud; Evaluación de programas y proyectos de salud.

\section{INTRODUÇÃO}

As ações de monitoramento e avaliação possuem objetivos intrinsecamente vinculados ao desafio de consolidação do Sistema Único de Saúde (SUS). Podem ser vistas também como intervenções para aumentar a capacidade de governo, com vistas à melhoria de seu projeto político, contribuindo, assim, para a governabilidade do sistema de saúde. ${ }^{1}$

A atividade de avaliar é conceituada como a aplicação de um julgamento de valor, através de dispositivos capazes de fornecer informações válidas e legítimas sobre a ação, permitindo aos envolvidos apresentar diferentes julgamentos. Esse julgamento pode ser o resultado da aplicação de um procedimento científico, como é o caso das pesquisas avaliativas. $^{2}$

A avaliação é um processo em que os avaliadores e interessados produzem, conjunta e colaborativamente, informações úteis sobre o objeto avaliado visando a sua qualificação. Há um longo percurso para se alcançar a institucionalização da avaliação em um sistema organizacional, que requer colaboração das instâncias, conquista de espaço político, autonomia dos recursos necessários e fortalecimento da capacidade avaliativa dos envolvidos. ${ }^{3}$

A necessidade de melhorar os processos de tomada de decisão está cada vez maior, desse modo, institucionalizar a avaliação pode ser uma alternativa ao sistema de saúde. A avaliação permite monitorar a capacidade dos serviços em responder às necessidades em saúde; acompanhar os efeitos das intervenções; identificar problemas e instrumentalizar equipes de saúde, gestores e comunidades. ${ }^{1}$

Assim, a institucionalização da avaliação representa uma estratégia importante a ser promovida para melhorar a eficiência do sistema de saúde, tendo em vista que a melhora do desempenho do sistema passa por uma melhora do processo de decisão. A pertinência da avaliação é maior quando há um grande número de atores envolvidos, fazendo com que seja verdadeiramente um condutor de mudanças. ${ }^{4}$

No Brasil, desde os anos 90, diversos esforços a fim de 


\section{ISSN 2236 - 1987}

institucionalizar a avaliação em saúde podem ser observados, inclusive 0 desenvolvimento de uma cultura avaliativa na Atenção Primária à Saúde (APS). Essa expansão e relativa autonomização da avaliação em saúde fez emergir debates sobre a sua constituição no interior do setor Saúde no país. ${ }^{5}$

As avaliações voltadas à implantação de programas e serviços de saúde vêm aumentando no Brasil, através da utilização de diferentes instrumentos e metodologias. Tem se destacado os estudos avaliativos no contexto da gestão hospitalar que envolvem os processos de Acreditação Hospitalar, visando replicar o papel dos chamados sistemas de certificação do controle da qualidade. Esses processos são voltados para a gestão $\mathrm{da}$ qualidade da assistência, aumento da eficiência e eficácia financeira, organização de processos, atendimento de requisitos dos pacientes, desenvolvimento e satisfação dos colaboradores nos ambientes hospitalares, além de melhorias na segurança do paciente. ${ }^{6-8}$

Já no contexto da APS, desafios ainda são enfrentados para satisfazer plenamente as necessidades dos usuários e para definir um novo modelo assistencial no país, a partir das propostas da Política Nacional da Atenção Básica. Diante da complexidade da gestão do Sistema Único de Saúde (SUS) é essencial que se faça uso dos instrumentos de avaliação dos serviços como ferramenta facilitadora de mudanças, pois a avaliação das características intrínsecas à APS pode direcionar qual o melhor percurso para a manutenção e/ou avanço na qualidade assistencial. ${ }^{9}$

No entanto, ainda que se destaque uma preocupação com os resultados dos processos avaliativos, é necessário que se extrapole o conhecimento gerado para os gestores dos programas, buscando influenciar a tomada de decisão. ${ }^{10}$

Nesse sentido, ganha relevância a compreensão da consolidação da avaliação de programas e políticas de APS no Brasil, bem como os usos que os resultados dessas avaliações possam ter para subsidiar os gestores e as equipes. Desse modo, o objetivo deste estudo é conhecer e sintetizar a produção científica sobre avaliação em saúde nos serviços de Atenção Primária à Saúde no Brasil.

\section{MATERIAIS E MÉTODOS}

Trata-se de uma revisão integrativa da literatura cujo método permite buscar, avaliar e sintetizar as evidências disponíveis para contribuir com 0 desenvolvimento do conhecimento da temática. ${ }^{11}$ As revisões integram uma diversidade de conhecimentos através do emprego de métodos rigorosos, que têm potencial para reunir os achados de estudos desenvolvidos mediante diferentes metodologias, contribuindo para 0 aprofundamento do tema investigado. ${ }^{12}$

Para a elaboração da revisão foram utilizadas as etapas indicadas para este tipo de pesquisa. As etapas adotadas foram: seleção do tema e elaboração da questão da pesquisa; estabelecimento de critérios de inclusão e exclusão dos estudos; 


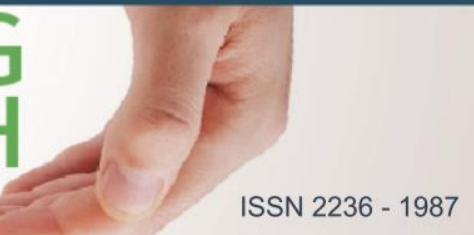

categorização

dos

estudos

selecionados e interpretação; análise crítica e discussão dos resultados e por fim, apresentação dos resultados encontrados. ${ }^{12-13}$

Para orientar o desenvolvimento do estudo, utilizou-se a seguinte questão de pesquisa: Qual a produção de conhecimento brasileira sobre avaliação em saúde nos serviços de Atenção Primária? Partindo dessa indagação, avançou-se para a etapa dos critérios de inclusão e exclusão e para a busca nas bases de dados.

A estratégia de identificação e seleção dos estudos foi a busca de publicações indexadas nas bases de dados Scientific Electronic Library Online (SciELO), Literatura LatinoAmericana e do Caribe em Ciências da Saúde (LILACS) e National Library of Medicine (MEDLINE).

Para a seleção dos artigos foram utilizados os seguintes critérios de inclusão: artigos brasileiros resultado de pesquisa e revisão de literatura, publicados entre os anos de 2010 e 2016, nos idiomas português, inglês e espanhol com acesso online e gratuito do texto completo. Os descritores em ciências da saúde (DeCS) utilizados para títulos e/ou resumos foram: Avaliação em Saúde e Atenção Primária à Saúde.

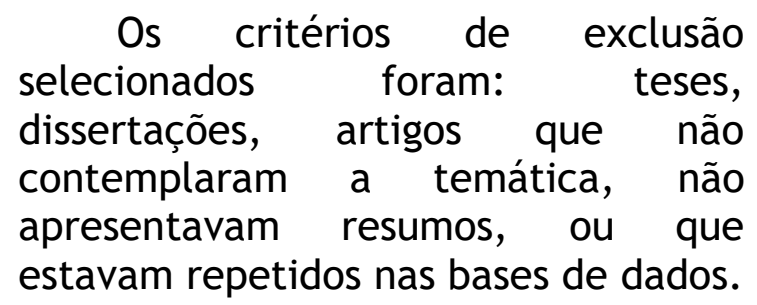

A busca das publicações ocorreu no mês de abril de 2017. Inicialmente, fez-se um levantamento nas bases
SCiELO, LILACS e MEDLINE utilizando os descritores Avaliação em Saúde e Atenção Primária à Saúde, nos idiomas escolhidos. Esta busca resultou em 135, 192 e 818 artigos, respectivamente, totalizando 1.145 artigos encontrados. Em seguida foi realizado um refinamento com a utilização dos critérios de inclusão e exclusão de publicações repetidas, que resultaram respectivamente, em seis, nove e oito produções, perfazendo nesta fase, 23 artigos.

$\mathrm{Na}$ sequência, realizou-se a leitura atenta dos títulos e dos resumos dos 23 artigos, sendo excluídos oito por não atenderem plenamente ao objetivo do estudo. Assim, foram selecionados para análise final 15 artigos que atenderam a todos os critérios e se adequaram ao objetivo deste estudo, os quais fizeram parte desta pesquisa.

Foi realizada a leitura minuciosa de cada um dos artigos obtidos na coleta de dados. Para a organização dos dados, as pesquisadoras elaboraram um instrumento de coleta de dados contendo: título, autor, periódico, ano de publicação, método de análise, objetivo e principais achados.

Para a classificação dos artigos por nível de evidência, foi empregado o sistema de classificação do nível de evidência de Melnyk e FineoutOverholt. ${ }^{14}$ Este sistema é composto por sete níveis, sendo: Nível I evidências originárias de revisões sistemáticas ou meta-análise de relevantes ensaios clínicos; Nível II evidências derivadas de pelo menos um ensaio clínico randomizado controlado bem delineado; Nível III - ensaios clínicos bem delineados sem randomização; Nível IV - estudos de 


\section{JOURNAIOF

coorte e de caso-controle bem delineados; Nível V - revisão sistemática de estudos descritivos e qualitativos; Nível VI - evidências derivadas de um único estudo descritivo ou qualitativo e Nível VII opinião de autoridades ou relatório de comitês de especialistas.

A análise de dados ocorreu de duas etapas. Na primeira etapa foi realizada a caracterização do perfil das publicações através das informações extraídas do roteiro de coleta de dados. Foram utilizados cálculos de frequência simples e relativa. $\mathrm{Na}$ segunda etapa procedeu-se a leitura detalhada das publicações e se realizou a análise do conteúdo dos artigos. Os dados foram tratados pela técnica de análise de conteúdo temática. ${ }^{15}$

\section{RESULTADOS E DISCUSSÃO}

\section{Caracterização dos estudos}

Foram encontrados 15 artigos $(100 \%)^{3,9,16-20,22,25-28,30,35,37}$ sobre a avaliação em saúde na Atenção Primária à Saúde no Brasil. 0 periódico que concentra o maior número de publicações é Cadernos de Saúde Pública, com quatro artigos $(26 \%)^{3,18,25,37}$, seguido por um artigo em cada uma das demais 11 revistas $(73 \%)^{9}, 16-17,19-20,22,26-28,30,35$ : $\quad$ Revista Brasileira de Educação Médica, Escola Anna Nery, Saúde em Debate, Revista Semina: Ciências Biológicas e da Saúde, Revista de Enfermagem do
Centro-Oeste Mineiro, Epidemiologia e Serviços de Saúde, Cadernos Saúde Coletiva, Revista Gaúcha de Enfermagem, Revista de Saúde Pública, General Hospital Psychiatry e Psychiatric Quarterly.

A maioria das publicações são dos anos de 2013, 2015 e 2016, com três artigos em cada ano, ou seja, nove publicações $(60 \%)^{9,16-20,22,27,30}$, demonstrando que a temática avaliação em saúde é uma discussão atual na literatura, apesar de em todos os anos pesquisados possuir ao menos uma publicação. Constataram-se duas publicações em $2010(13 \%)^{35,37}$, duas em $2012(13 \%)^{26,28}$, seguido de uma em $2011(6 \%)^{25}$ e uma em $2014(6 \%)^{3}$.

Dos artigos integrantes da pesquisa, $11(73 \%)^{3,9,16-20,22,27-28,30}$ se caracterizam por sua natureza quantitativa e seis $(40 \%)^{16,18,22,25-26,37}$ se caracterizam como pesquisas avaliativas. Somente dois artigos $(13 \%)^{26,35}$ são qualitativos. Uma parte expressiva, ou seja, sete $(46 \%)^{9,16-20,30}$ dos estudos selecionados utilizam em suas avaliações o Instrumento de Avaliação da Atenção Primária denominado PCATool-Brasil, que visa dimensionar a extensão dos atributos essenciais e derivados da APS. Analisando-se o nível de evidência, 13 artigos $(86 \%)^{9,16-20,22,25-28,30,35}$ foram classificados com nível de evidência fraco (VI). Um $(6 \%)^{3}$ com forte nível de evidência (I) e um $(6 \%)^{37}$ com nível IV. Apresenta-se na figura 1, a seguir, uma síntese dos artigos selecionados para esta revisão. 
Figura 1. Dados dos artigos selecionados. Porto Alegre - RS, 2017.

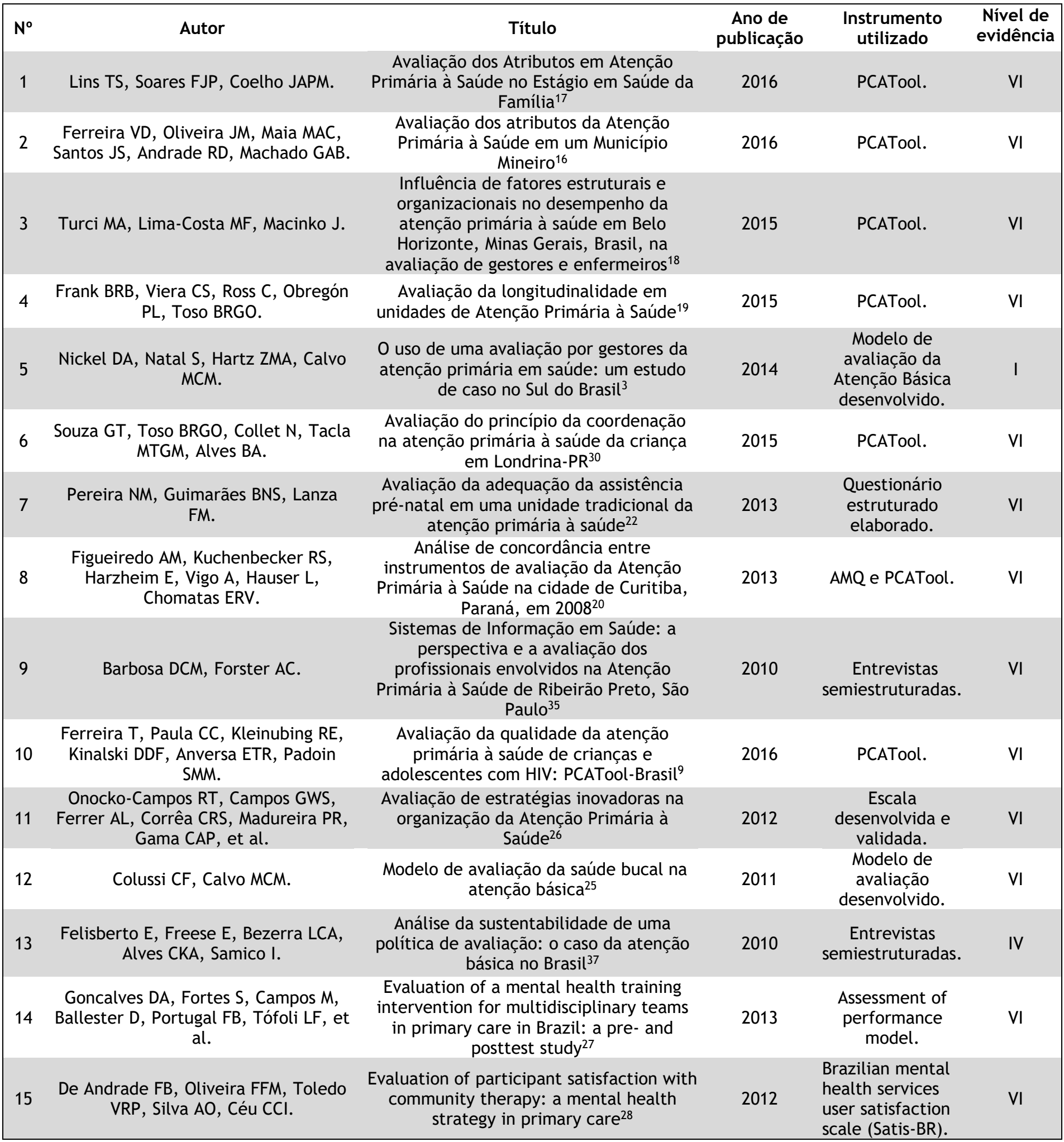




\section{IOURNAIOF

A fim de possibilitar a discussão e seguindo a metodologia adotada, categorias temáticas que emergiram foram: Uso de instrumentos para Avaliação em Saúde e Avaliação do desempenho da Atenção Primária à Saúde.

\section{Uso de instrumentos para Avaliação em Saúde}

Sobre o uso de instrumentos para a avaliação em saúde na atenção primária foram encontrados 10 artigos. Objetivando a qualificação da gestão e da assistência de saúde, os estudos encontrados. ${ }^{9,16-20,30}$ utilizaram em suas avaliações sobre o desempenho dos serviços, o instrumento de avaliação da atenção primária Primary Health Care Assessment Tool (PCATool), desenvolvido por Starfield e Shi e validado no Brasil pelo Ministério da Saúde.

Nesta perspectiva, é apontado na literatura que esse instrumento é capaz de mensurar a adequação dos atributos essenciais e derivados da APS nos serviços de saúde, bem como avaliar a qualidade e a capacidade de respostas frente às diferentes situações em saúde na APS. ${ }^{16}$

A partir dos resultados de outro estudo $^{17}$ que avaliou os níveis de cuidados de saúde primários prestados por preceptores de estágio, evidenciou-se que o PCATool também pode ser utilizado como uma ferramenta na integração ensinoserviço. A existência de padrões nacionais desse instrumento permitiria a avaliação da eficácia e a implementação de planos e ajustes nas intervenções educativas.
Foram encontrados estudos $9,18-19$ que apontam o PCATool como um bom instrumento para avaliação do desempenho da APS em diferentes contextos. Destacam a sua utilização como uma ferramenta efetiva para avaliar os serviços de APS, uma vez que é um questionário amplamente validado. Por contemplar todos os atributos da APS, esse instrumento possibilita identificar a sua extensão nos serviços avaliados, bem como reconhecer os aspectos frágeis de cada um, para que se possa intervir e reorganizar a atenção à saúde de maneira mais efetiva e direcionada. ${ }^{19}$

Outro estudo ${ }^{20}$ analisou a concordância entre os instrumentos PCATool e AMQ-AB (Avaliação para Melhoria da Qualidade da Atenção Básica) - elaborado pelo Ministério da Saúde. Os resultados sugeriram não ser possível a utilização dos padrões do AMQ como forma de mensurar todos os atributos da APS, diferentemente do PCATool, já validado nacionalmente para esse fim. Foi apontado que houve concordância dos dois instrumentos para os atributos 'integralidade' e 'orientação familiar', o que não ocorreu em relação aos demais atributos da APS.

Em relação ao instrumento do $A M Q-A B$, a literatura aponta que não foi delineado de forma a avaliar todos os componentes da APS, não permitindo dessa forma, avaliar como as ações estão sendo implementadas e de que maneira elas são efetivas, gerando os resultados desejados. ${ }^{21}$

Por sua vez, um estudo ${ }^{3}$ que buscou identificar o uso e os fatores da capacidade avaliativa em gestores de um estado do Sul do Brasil, 


\section{ISSN 2236 - 1987}

desenvolveu um de pronta resposta para gestores. Entre os resultados, apontou-se que modelo de avaliação da atenção básica o uso do instrumento para planejamento de ações ocorreu no município de menor porte populacional e quanto menor a apropriação do processo, menor a chance de uso.

Para avaliar a qualidade da assistência pré-natal, um estudo ${ }^{22}$ elaborou um questionário estruturado, tendo como marco teórico o manual do Ministério da Saúde sobre Pré-Natal de Baixo Risco, cujos resultados demonstraram uma inadequação da assistência a essa situação de saúde.

Discutindo a avaliação do cuidado pré-natal, estudos apontam que evidências, cientificamente embasadas, permitem aos profissionais e gestores, a implementação de medidas para melhorar a qualidade da assistência e reorganizar a prestação desse atendimento na atenção primária. $23-24$

Buscando apresentar um modelo para avaliar a qualidade da atenção básica em saúde bucal e testar sua aplicabilidade em municípios de diferentes portes em SC, um trabalho ${ }^{25}$ desenvolveu um modelo de avaliação contendo duas dimensões com seus indicadores e medidas. Nos resultados, o modelo mostrou-se aplicável e de grande utilidade para municípios de diferentes portes, possibilitando a identificação dos pontos nos quais poderiam ser aperfeiçoadas as ações em saúde bucal.

Já para comparar o desempenho de Unidades Básicas de Saúde segundo a implantação de novos arranjos e estratégias de atenção primária e saúde mental, foi realizado um estudo avaliativo $^{26}$, qualitativo, que desenvolveu uma escala de avaliação. Concluiu-se após a avaliação, a necessidade de promover a organização da assistência em saúde mental nas Unidades Básicas de Saúde (UBS), implantando avaliações de risco e dispositivos de intervenção oportunos para esses transtornos. Outros estudos ${ }^{27-28}$ que avaliaram a saúde mental na atenção primária, utilizaram instrumentos de avaliação do desempenho e ambos apontaram a necessidade de mudanças mais amplas no sistema de cuidados em saúde mental.

Com relação à discussão dos instrumentos e modelos de avaliação, um estudo procurou o instrumento "mais adequado" para avaliar a APS. Demonstrou que o principal objetivo nas pesquisas de avaliação é a produção de um conhecimento que seja reconhecido pela comunidade cientifica e que sirva como fator orientador de decisão. Os resultados advindos da aplicação de um instrumento de pesquisa devem dar subsídios para a criação de novas propostas que possam contribuir para a qualificação da APS. Evidenciou-se que para avaliar a Saúde da Família o instrumento que mais se aproxima da proposta é o PCATool. ${ }^{29}$

Também foram encontrados estudos ${ }^{18-19}$ que evidenciam que ainda há muito a se avançar na avaliação dos serviços de saúde em território nacional, inclusive no sentido de proporcionar comparações regionais entre os desempenhos da APS, a fim de se aperfeiçoar a atenção prestada em 


\section{JOURNALOF \\ NURSING \\ ANO HEALTH}

ISSN 2236 - 1987

fortemente no seu desempenho, apontando a necessidade de investimentos em Unidades Básicas de Saúde (UBS) de porte médio, robustas e bem equipadas, que possibilitem a permanência de várias equipes de Saúde da Família.

Outro estudo ${ }^{30}$ avaliou o princípio da coordenação da APS com relação à saúde da criança em Unidades Básicas de Saúde de um município do Paraná (PR), através do PCATool-Brasil, versão criança. Observaram-se altos escores do atributo coordenação na APS em seus resultados, ou seja, segundo a percepção dos usuários entrevistados o atributo é capaz de exercer sua função. No entanto, evidenciou-se a fragilidade de alguns itens na atenção primária à saúde da criança, principalmente relativo ao cuidado integral no serviço especializado.

A partir dos resultados de um estudo $^{19}$ que avaliou a longitudinalidade do cuidado na APS em um município do estado do PR, através do PCATool, identificou-se que para cuidadores de crianças, esse atributo foi mais bem avaliado na UBS sem ESF do que na UBS com ESF. Apontam como razões a não ocorrência de mudanças no modelo de atenção da ESF e as práticas de saúde ainda frágeis, reforçando o modelo assistencial biomédico. Apontou-se a necessidade de mudanças profundas no processo de trabalho das ESF, apesar de não ter sido possível observar o papel da APS em sua total abrangência no referido estudo.

Em contrapartida, outro estudo ${ }^{16}$ avaliou a extensão dos atributos da APS na perspectiva dos enfermeiros das ESF e das UBS convencionais em um 
ISSN 2236 - 1987

município do estado de Minas Gerais (MG), utilizando o PCATool-Brasil, versão profissionais. Verificou-se que a ESF apresentou maiores escores em todos os atributos, com exceção do acesso de primeiro contato. 0 atributo integração de cuidados apresentou a menor diferença entre UBS e ESF, enquanto a orientação comunitária recebeu a maior diferença entre as unidades. Assim, concluiu-se que a ESF atingiu melhores resultados nos atributos da APS em relação às UBS convencionais, todavia foi destacada a necessidade de aperfeiçoamentos no processo de trabalho das equipes.

No mesmo sentido, um estudo quantitativo 9 avaliou o escore geral da APS nos municípios de residência de crianças e adolescentes com HIV, através do PCATool-Brasil, versão profissional. Nos resultados, a avaliação geral da qualidade dos serviços de UBS e ESF foi satisfatória, mas quando comparados, somente a ESF apresentou um alto escore.

Corroborando com os resultados das duas pesquisas anteriores, um estudo $^{17}$ que avaliou os atributos da APS por meio do PCATool-Brasil aplicado aos preceptores médicos das ESF em Alagoas (AL), evidenciou fragilidades nos atributos de acesso, coordenação do cuidado e integralidade. As ESF de ensino avaliadas apresentaram, em sua maioria, escores satisfatórios dos atributos da APS de maneira a contribuir para uma formação adequada às necessidades de saúde da população e o aprimoramento da integração ensino-serviço.

Discutindo a qualidade da APS, a literatura aponta um melhor desempenho das unidades com ESF em relação aos atributos longitudinalidade, integralidade dos serviços prestados, orientação comunitária e consequentemente no escore geral em relação às unidades sem ESF. Desse modo, é apontado que o processo de trabalho das ESF favorece que tais atributos apresentem melhor desempenho, sendo esse o modelo que agrega mais qualidade ao desempenho da APS. ${ }^{31}$

Com relação ao desempenho das equipes de APS na atenção prestada a situações específicas de saúde, alguns estudos ${ }^{9,22,25-26}$ trazem a avaliação como método para monitorar os resultados em relação à organização e provisão dos serviços/programas, a fim de orientar a reorganização das ações para uma atenção à saúde de qualidade.

Para avaliar a adequação da assistência pré-natal, um estudo quantitativo $^{22}$ avaliou as unidades tradicionais da APS de um município mineiro. Concluiu-se que a assistência pré-natal no local, em seus componentes mais básicos, apresentou inadequações. Sugeriu-se a qualificação dos profissionais para melhoria da qualidade da assistência pré-natal.

Quanto à qualificação do prénatal, a literatura aponta a necessidade de sensibilizar todos os profissionais de saúde, tanto aqueles que atuam na ESF quanto os que atuam em unidades de saúde tradicionais. Ressalta-se a importância das equipes seguirem os protocolos recomendados e a padronização dos cuidados à gestante, de forma a garantir uma boa qualidade da atenção pré-natal. ${ }^{32}$ 
Quanto à avaliação das estratégias de saúde mental na atenção primária, uma pesquisa avaliativa ${ }^{26}$ comparou as unidades básicas de saúde de um município do estado de São Paulo (SP). Foram identificados avanços positivos através da integração dos agentes comunitários nas equipes. Dificuldades foram identificadas na comunicação entre os níveis de atenção e na implantação do apoio matricial.

Ainda nessa temática, estudos publicados em revistas americanas ${ }^{27-28}$ também avaliaram o desempenho da saúde mental na atenção primária. Um dos estudos ${ }^{27}$ avaliou uma intervenção para melhorar a assistência de saúde mental nos cuidados primários, concluindo que a formação dos profissionais em um modelo de cuidado compartilhado, não se associou com melhorias consistentes no reconhecimento ou na gestão dos problemas de saúde mental. Já o outro estudo ${ }^{28}$ avaliou a satisfação dos usuários com relação a Terapia Comunitária na atenção primária, que evidenciou através de avaliações positivas, ser uma importante ferramenta de saúde mental na atenção primária.

Nessa perspectiva, a qualificação clínica da rede básica para a atenção à saúde mental, com a participação de equipes de apoio matricial, foi citada em um estudo como forma de orientar a produção de saúde. A construção de projeto terapêutico singular para usuários também foi um tema abordado, principalmente nos municípios de grande porte populacional. 33
Já para avaliar a qualidade da saúde bucal na atenção primária em Santa Catarina (SC), foi utilizado em um estudo ${ }^{25}$, um modelo com duas dimensões. Os critérios de efetividade e eficácia evidenciaram que o município deve facilitar o acesso aos serviços por meio de mecanismos ágeis de agendamento, garantindo atenção integral, resolutiva e individualizada, bem como a necessidade de melhoria na qualidade do registro dos dados. Desse modo, busca-se promover a melhoria dos serviços, e consequentemente, da saúde da população.

Corroborando com esses resultados, foi encontrado na literatura um estudo que verificou um impacto positivo dos indicadores "acesso" e "rendimento" após a implementação da saúde bucal na ESF. Os autores relacionaram os resultados ao aumento do número de profissionais e ao amadurecimento do processo de trabalho. ${ }^{34}$

Em relação à avaliação da percepção dos profissionais da ESF sobre o Sistema de Informação em Saúde que manipulam, foi identificado em um estudo ${ }^{35}$ que o uso cotidiano do sistema não alcançou adequadamente o objetivo de ser um instrumento de reorganização das práticas de trabalho. Mesmo com as dificuldades, concluiu-se que um sistema de informação deve ser a base de dados fundamental da APS para o planejamento local das ações de saúde.

Nesse sentido, a busca por resultados diferentes no trabalho implica em uma boa gestão dos 
sistemas de informações, planejamento, monitoramento e avaliação das intervenções. Ou seja, as equipes têm de aprender a trabalhar com as tecnologias de que dispõe para usá-las da maneira mais eficiente possível. ${ }^{33}$

Discutindo o uso da avaliação em programas e serviços na atenção primária, a literatura aponta que a compreensão da dinâmica de desenvolvimento das práticas de atenção ao usuário parece essencial para desvendar as especificidades relacionadas às dificuldades de êxito das ações implementadas no âmbito desse nível de atenção. ${ }^{36}$

Os processos avaliativos tendem a abordar mais de um aspecto dos programas avaliados, numa perspectiva de análise mais abrangente. A questão em torno do quanto e como os programas e serviços influenciam as áreas a que eram destinados emerge enquanto uma preocupação constante dos processos avaliativos. ${ }^{10}$

Portanto, a avaliação das políticas públicas de saúde permite a produção de informações com vistas à melhoria da eficácia do sistema, bem como a possibilidade de transformação das práticas de atenção. A prática avaliativa necessita de certo grau de institucionalização para que se torne efetiva e produza efeitos formativos sobre a conduta da ação pública. ${ }^{37}$

\section{CONSIDERAÇÕES FINAIS}

Os estudos analisados nesta revisão permitiram conhecer e sintetizar as evidências disponíveis na literatura, entre o período de 2010 a 2016, relacionadas à avaliação em saúde nos serviços de Atenção Primária no Brasil.

Constatou-se que a avaliação em saúde é essencial para mensurar o desempenho do sistema de saúde, sendo uma ferramenta condutora de mudanças nos processos de atenção à saúde. Quanto ao instrumento utilizado para avaliar, observou-se que há predominância do PCATool, validado nacionalmente. Esse instrumento abrange tanto a avaliação dos atributos essenciais e derivados da APS, como identifica os aspectos a serem melhorados, para que se possa reorganizar a atenção à saúde.

Outros instrumentos, como os modelos de avaliação de desempenho desenvolvidos por pesquisadores, também podem ser úteis para a avaliação em saúde. Esses modelos devem possibilitar a avaliação do acesso e da utilização dos serviços da APS, bem como dos resultados em saúde.

Destaca-se a Estratégia Saúde da Família como o modelo que apresenta melhores resultados quanto os atributos da APS e o desempenho geral da qualidade da atenção, em relação às unidades básicas de saúde convencionais ou outros modelos de equipes. Entretanto, foi evidenciada a necessidade de aperfeiçoamento no processo de trabalho da ESF, sobretudo na assistência ao pré-natal, à saúde mental, à saúde bucal e na utilização dos sistemas de informação.

Os achados deste estudo, também apontaram evidência fraca nos artigos selecionados. Apenas um deles apresentou evidência de nível I. É necessário que sejam realizados 


\section{JOURNALOF \\ NURSING \\ MOHEALTH}

ISSN 2236 - 1987

estudos de maior evidência, a fim de orientarem a prática de avaliação em saúde na atenção primária.

Salienta-se

que

a

institucionalização da avaliação em saúde pode ser efetivada a partir do aprimoramento e da utilização de instrumentos e métodos que promovam a reorganização das práticas de atenção à saúde. As contribuições do estudo, no que se refere à avaliação em saúde na APS, implicam nos usos que os resultados dessas avaliações possam ter para subsidiar os gestores em suas tomadas de decisão e as equipes em seus planejamentos da assistência à saúde, bem como o monitoramento constante dos resultados.

Aponta-se como sugestão, a realização de avaliações em saúde na atenção primária e de publicações sobre a temática, de modo a embasar outros estudos e orientar os gestores e os profissionais de saúde. Promovendo, assim, a mensuração dos processos de atenção à saúde, a qualificação do desempenho do sistema, e consequentemente, impactos na melhoria da saúde da população.

\section{REFERÊNCIAS}

1. Carvalho ALB, Souza MF, Shimizu HE, Senra IMVB, Oliveira KC. A gestão do SUS e as práticas de monitoramento e avaliação: possibilidades e desafios para a construção de uma agenda estratégica. Cienc saude coletiva [Internet]. 2012 abr[acesso em 2017 abr 24];17(4):901-11. Disponível em: http://www.scielo.br/scielo.php?scrip $\mathrm{t}=\mathrm{sci}$ arttext\&pid=S1413-

81232012000400012
2. Contandriopoulos AP, Pouvourville G, Poullier JP, Contandriopoulos D. À la recherche d'une troisième voie: les systèmes de santé au XXI e siècle. In: Pomey MP, Poullier JP, éditeurs. Santé publique; 2000 [acesso em 2017 jan 18];р.637-67.

3. Nickel DA, Natal S, Hartz ZMA, Calvo MCM. O uso de uma avaliação por gestores da atenção primária em saúde: um estudo de caso no Sul do Brasil. Cad saúde pública [Internet]. 2014 dez[acesso em 2017 jan 18];30(12):2619-30. Disponível em: http://www.scielo.br/scielo.php?scrip $\mathrm{t}=\mathrm{sci}$ _arttext\&pid=S0102-

311X2014001202619\&lng=en

4. Contandriopoulos AP. Avaliando a institucionalização da avaliação. Cienc saude colet [Internet]. 2006 set[acesso em 2017 jan 18];11(3):705-711. Disponível em: http: / / www.scielo.br/scielo.php?scrip $\mathrm{t}=\mathrm{sci}$ arttext\&pid=S1413-

$81232006000300017 \& \operatorname{lng}=$ en

5. Furtado JP, Vieira-da-Silva LM. A avaliação de programas e serviços de saúde no Brasil enquanto espaço de saberes e práticas. Cad saude publica [Internet]. $2014 \mathrm{dez}$ [acesso em 2017 abr 15];30(12):2643-55. Disponível em: http: / / www.scielo.br/scielo.php?scrip $\mathrm{t}=\mathrm{sci}$ arttext\&pid=S0102-

$311 \times 2014001202643 \& \operatorname{lng}=e n$

6. Jorge MJ, Carvalho FA, Sales PR. Determinantes organizacionais do ambiente hospitalar: uma análise empírica usando microdados sobre conformidade segundo o modelo da Acreditação Hospitalar. Revista de administração hospitalar [Internet]. 2014 jan/mar[acesso em 2017 abr 


\section{JOURNALOF \\ NURSING \\ AND HEALTH}

15];11(1):68-82. Disponível em: http://revistas.face.ufmg.br/index.ph $\mathrm{p} / \mathrm{rahis} /$ article/view/1949/1312

7. Siman AG, Brito MJM, Carrasco MEL. Participação do enfermeiro gerente no processo de acreditação hospitalar. Rev gaucha enferm [Internet]. 2014 jun[acesso em 2017 abr 15];35(2):93-9. Disponível

em:

http: / / www.scielo.br/scielo.php?scrip $\mathrm{t}=\mathrm{sci}$ arttext\&pid=S1983-

$14472014000200093 \& \operatorname{lng}=e n$

8. Siman AG, Cunha SGS, Brito MJM. Mudanças nas ações gerenciais após a Acreditação Hospitalar. Rev rene [Internet]. $2016 \mathrm{mar} / \mathrm{abr}$ [acesso em 2017 jan 18];17(2):165-75. Disponível em:

http://periodicos.ufc.br/rene/article /view/2992/2308

9. Ferreira T, Paula CC, Kleinubing RE, Kinalski DDF, Anversa ETR, Padoin SMM. Avaliação da qualidade da atenção primária à saúde de crianças e adolescentes com HIV: PCATool-Brasil. Rev gauch enferm [Internet]. 2016 ago[acesso em 2017 jan 18];37(3):e61132. Disponível em: http://www.scielo.br/scielo.php?scrip $\mathrm{t}=\mathrm{sci}$ arttext\&pid=S1983-

$14472016000300407 \& \operatorname{lng}=e n$

10. Gasparini MFV, Furtado JP. Avaliação de Programas e Serviços Sociais no Brasil: uma análise das práticas no contexto atual. Serv soc soc. 2014 jan/mar[acesso em 2017 jan 18];117:122-41.

11. De Souza MT, Da Silva MD, De Carvalho R. Revisão integrativa: o que é e como fazer. Rev einstein. 2010 [acesso em 2017 abr 15];8(1):102-6.

12. Soares CB, Hoga LAK, Peduzzi M, Sangaleti C, Yonekura T, Silva DRAD.
ISSN 2236 - 1987

Revisão integrativa: conceitos e métodos utilizados na enfermagem. Rev esc enferm [Internet]. 2014 abr[acesso em 2017 abr 15];48(2):335-45. Disponível em: http://www.scielo.br/scielo.php?scrip $\mathrm{t}=\mathrm{sci}$ arttext\&pid=S0080-

62342014000200335\&lng=en

13. Mendes KDS, Silveira RCCP, Galvão CM. Revisão integrativa: método de pesquisa para a incorporação de evidências na saúde e na enfermagem. Texto \& contexto enferm [Internet]. 2008 [acesso em 2017 abr 17];17(4):758-64. Disponível em: http://www.scielo.br/scielo.php?scrip $\mathrm{t}=\mathrm{sci}$ _arttext\&pid=S010407072008000400018.

14. Melnik BM, Fineout-Overholt E. Making the case for evidence-based practice and cultivating a spirit of inquiry. In: Melnyk BM, FineoutOverholt $\mathrm{E}$. Evidence-based practice in nursing \& healthcare. A guide to best practice.

Philadelphia: Lippincott Williams \& Wilkins; 2005.

15. Minayo MCS. O desafio do conhecimento: pesquisa qualitativa em saúde. $12^{a}$ ed. São Paulo: Hucitec; 2010.

16. Ferreira VD, Oliveira JM, Maia MAC, Santos JS, Andrade RD, Machado GAB. Avaliação dos atributos da Atenção Primária à Saúde em um Município Mineiro. Esc Anna Nery [Internet]. 2016 [acesso em $2017 \quad \mathrm{abr}$ 17];20(4):e20160104. Disponível em: http://www.scielo.br/scielo.php?scrip $\mathrm{t}=\mathrm{sci}$ _arttext\&pid=S1414-

$81452016000400221 \& \operatorname{lng}=e n$

17. Lins TS, Soares FJP, Coelho JAPM. Avaliação dos Atributos em Atenção Primária à Saúde no Estágio em Saúde 
da Família. Rev bras educ med [Internet]. 2016 set[acesso em 2017 jan 18];40(3):355-63. Disponível em: http: //www.scielo.br/scielo.php?scrip $\mathrm{t}=\mathrm{sci}$ arttext\&pid=S0100-

$55022016000300355 \& \operatorname{lng}=e n \& n r m=$ iso

18. Turci MA, Lima-Costa MF, Macinko J. Influência de fatores estruturais e organizacionais no desempenho da atenção primária à saúde em Belo Horizonte, Minas Gerais, Brasil, na avaliação de gestores e enfermeiros. Cad saude publica [Internet]. 2015 set[acesso em 2017 jan 18];31(9):1941 52. Disponível em: http://www.scielo.br/scielo.php?scrip $\mathrm{t}=\mathrm{sci}$ _arttext\&pid=S0102.

$311 X 2015000901941 \& \operatorname{lng}=e n$

19. Frank BRB, Viera CS, Ross C, Obregón PL, Toso BRGO. Avaliação da longitudinalidade em unidades de Atenção Primária à Saúde. Saúde debate online [Internet]. 2015 jun[acesso em 2017 jan 18];39(105):400-10. Disponível em: http://www.scielo.br/scielo.php?scrip $\mathrm{t}=\mathrm{sci}$ arttext\&pid=S0103-

$11042015000200400 \& \operatorname{lng}=\mathrm{en}$

20. Figueiredo AM, Kuchenbecker RS, Harzheim E, Vigo A, Hauser L, Chomatas ERV. Análise de concordância entre instrumentos de avaliação da Atenção Primária à Saúde na cidade de Curitiba, Paraná, em 2008. Epidemiol serv saúde. 2013 jan/mar[acesso em 2017 jan 25];22(1):41-8.

21. Teixeira MB, Casanova A, Oliveira CCM, Engstrom EM, Bodstein RCA. Avaliação das práticas de promoção da saúde: um olhar das equipes participantes do Programa Nacional de Melhoria do Acesso e da Qualidade da
ISSN 2236 - 1987

Atenção Básica. Saude debate [Internet]. 2014 out[acesso em 2017 abr 17];38(n.esp.):52-68. Disponível em: http: / /www.scielo.br/scielo.php? script=sci_arttext\&pid=S0103. $11042014000600052 \&$ lng $=e n$

22. Pereira NM, Guimarães BNS, Lanza FM. Avaliação da adequação da assistência pré-natal em uma unidade tradicional da atenção primária à saúde. Rev enferm Cent-Oeste Min [Internet]. 2013 set/dez[acesso em 2017 jan 25];3(3):804-19. Disponível em:

http: / /www.seer.ufsj.edu.br/index.p $\mathrm{hp} / \mathrm{recom} /$ article/view/405

23. Zanchi M, Gonçalves CV, Cesar JÁ, Dumith SC. Concordância entre informações do Cartão da Gestante e do recordatório materno entre puérperas de uma cidade brasileira de médio porte. Cad saude publica [Internet]. 2013 mai[acesso em 2017 abr 17];29(5):1019-28. Disponível em: http: //www.scielo.br/scielo.php?scrip $\mathrm{t}=\mathrm{sci}$ _arttext\&pid=S0102-

311X2013000500019\&lng=en

24. Silva EP, Lima RT, Ferreira NLS, Costa MJC. Pré-natal na atenção primária do município de João PessoaPB: caracterização de serviços e usuárias. Rev bras saude mater infant [Internet]. 2013 mar[acesso em 2017 jan 25];13(1):29-37. Disponível em: http://www.scielo.br/scielo.php?scrip $\mathrm{t}=\mathrm{sci}$ _arttext\&pid=S1519-

$38292013000100004 \& \operatorname{lng}=\mathrm{en}$

25. Colussi CF, Calvo MCM. Modelo de avaliação da saúde bucal na atenção básica. Cad saude publica [Internet]. 2011 set[acesso em 2017 abr 17];27(9):1731-45. Disponível em: http://www.scielo.br/scielo.php?scrip 


\section{NURSING \\ aNo HEALTH}

ISSN 2236 - 1987

$\mathrm{t}=\mathrm{sci}$ arttext\&pid=S0102-

311X2011000900007\&lng=en

26. Onocko-Campos RT, Campos GWS, Ferrer AL, Corrêa CRS, Madureira PR, Gama CAP, et al. Evaluation of innovative strategies in the organization of Primary Health Care. Rev saude publica [Internet]. 2012 fev[acesso em 2017 jan 25];46(1):4350. Disponível em: http://www.scielo.br/scielo.php?scrip $\mathrm{t}=\mathrm{sci}$ arttext\&pid=S003489102012000100006\&lng=en

27. Goncalves DA, Fortes S, Campos M, Ballester D, Portugal FB, Tófoli LF, et al. Evaluation of a mental health training intervention for multidisciplinary teams in primary care in Brazil: a pre- and posttest study. Gen hosp psychiatry [Internet]. 2013 mai/jun[acesso em 2017 jan 18];35(3):304-8. Disponível em: https://www.ncbi.nlm.nih.gov/pubm ed/23521815

28. De Andrade FB, Oliveira FFM, Vianna RPT, Silva AO, Céu CCl. Evaluation of participant satisfaction with community therapy: a mental health strategy in primary care. Psychiatr Q [Internet]. 2012 set[acesso em 2017 jan 18];83(3):325-34. Disponível em: https://www.ncbi.nlm.nih.gov/pubm ed/22318711

29. Fracolli LA, Gomes MFP, Nabão FRZ, Santos MS, Cappellini VK, Almeida ACC. Instrumentos de avaliação da Atenção Primária à Saúde: revisão de literatura e metassíntese. Ciênc saúde coletiva [Internet]. 2014 dez[acesso em 2017 jan 18];19(12):4851-60. Disponível em: http://www.scielo.br/scielo.php?scrip $\mathrm{t}=\mathrm{sci}$ arttext\&pid=S1413$81232014001204851 \& \operatorname{lng}=$ en

30. Souza GT, Toso BRGO, Collet N, Tacla MTGM, Alves BA. Avaliação do princípio da coordenação na atenção primária à saúde da criança em Londrina-PR. Semina cienc biol saude [Internet]. 2015 jan/jun[acesso em 2017 abr 17];36(1):39-46. Disponível em:

http://www.uel.br/revistas/uel/inde x.php/seminabio/article/view/18420

31. Martins JS, Abreu SCC, Quevedo $M P$, Bourget MMM. Estudo comparativo entre Unidades de Saúde com e sem Estratégia Saúde da Família por meio do PCATool. Rev bras med fam comunidade. 2016 jan/dez[acesso em 2017 abr 19];11(38):1-13.

32. Mendoza-Sassi RA, Cesar JA, Teixeira TP, Ravache C, Araújo GD, Silva TC. Diferenças no processo de atenção ao pré-natal entre unidades da Estratégia Saúde da Família e unidades tradicionais em um município da Região Sul do Brasil. Cad saúde publica [Internet]. 2011 abr[acesso em 2017 abr 19];27(4):787-96. Disponível em: http://www.scielo.br/scielo.php?scrip $\mathrm{t}=\mathrm{sci} \_$arttext\&pid=S0102-

311X2011000400018\&lng=en

33. Cruz MM, Souza RBC, Torres RMC, Abreu DMF, Reis AC; Gonçalves AL. Usos do planejamento e autoavaliação nos processos de trabalho das equipes de Saúde da Família na Atenção Básica. Saúde debate [Internet]. 2014 out[acesso em 2017 jan 18];38(n.esp.):124-39. Disponível em: http://www.scielo.br/scielo.php?scrip $\mathrm{t}=\mathrm{sci}$ arttext\&pid=S010311042014000600124\&lng=en 


\section{NURSING \\ AND HEALTH}

34. Palacio DC, Vazquez FL, Ramos DVR, Peres SV, Pereira AC, Guerra LM, et al. Evolução de indicadores pósimplantação da saúde bucal na Estratégia Saúde da Família. Einstein [Internet]. 2014 mar[acesso em 2017 jan 25];12(3):274-81. Disponível em: http://www.scielo.br/scielo.php?scrip $\mathrm{t}=\mathrm{sci}$ arttext\&pid=S1679-

45082014000300274\&lng=en

35. Barbosa DCM, Forster AC. Sistemas de Informação em Saúde: a perspectiva e a avaliação dos profissionais envolvidos na Atenção Primária à Saúde de Ribeirão Preto, São Paulo. Cad saúde colet [Internet]. 2010 jul/set[acesso em 2017 abr 17];18(3):424-33. Disponível em: http://www.cadernos.iesc.ufrj.br/ca dernos/images/csc/2010_3/artigos/CS Cv18n3_pag424-33.pdf

36. Carvalho Filha FSS, Nogueira LT, Medina MG. Avaliação do controle de hipertensão e diabetes na Atenção Básica: perspectiva de profissionais e usuários. Saude debate. 2014 out[acesso em 2017 abr 17];38(n.esp.):265-78.

37. Felisberto $E$, Freese $E$, Bezerra LCA, Alves CKA, Samico I. Análise da sustentabilidade de uma política de avaliação: o caso da atenção básica no Brasil. Cad saúde publica [Internet]. 2010 jun[acesso em 2017 abr 19];26(6):1079-95. Disponível em: http://www.scielo.br/scielo.php?scrip $\mathrm{t}=\mathrm{sci} \_$arttext\&pid=S0102$311 \times 2010000600003 \& \operatorname{lng}=\mathrm{en}$

Data de submissão: 12/10/2017

Data de aceite: 26/02/2018

Data de publicação: 13/05/2018 